\title{
Adhesive root hairs facilitate Posidonia oceanica seedling settlement on rocky substrates
}

\author{
Alagna Adriana ${ }^{1}$, Vega Fernández Tomás ${ }^{1}$, D’Anna Giovanni', Fici Silvio², Magliola \\ Carlo $^{3}$, Badalamenti Fabio ${ }^{1}$ \\ ${ }^{1}$ CNR-IAMC, Istituto per l'Ambiente Marino Costiero, Castellammare del Golfo, Italy \\ 2 Dipartimento di Scienze Agrarie e Forestali, Università di Palermo, Italy \\ ${ }^{3}$ Saipem S.p.A., San Donato Milanese, Italy \\ *adriana.alagna@iamc.cnr.it
}

Posidonia oceanica, the dominant Mediterranean seagrass, has been historically described as a species typically growing on mobile substrates whose development requires precursor communities. During more than 10 years of direct observations, we noticed that $P$. oceanica seedlings were often firmly anchored to rocky reefs, even at exposed sites. Thus, we analysed the ultrastructural features of seedling root systems to identify specific traits that may represent adaptations for early seedling anchorage on rocky bottoms.

Subapical sections of adventitious roots were obtained from 2-3 months old specimens collected in the field and were observed at SEM revealing an extensive coverage of adhesive root hairs with a maximum length of $2400 \mu \mathrm{m}$. Hairs were provided with an enlarged tips with a maximum width of $78.3 \mu \mathrm{m}$, which extended the contact area between the hair tip and the substrate.

To test whether adhesive root hairs may facilitate $P$. oceanica seedlings establishment on rocky substrates, a manipulative experiment was performed. 360 seedlings were reared for 5 months in a land-based culture facility under simulated natural hydrodynamic conditions to identify suitable substrates for early seedling anchorage. Two main substrate features were investigated: firmness (i.e., sand vs. rock) and complexity (i.e., size of interstitial spaces between rocks). Anchorage was strongly influenced by substrate firmness and occurred only on rocks through adhesion by sticky root hairs. Percentage of anchored seedlings on rocks was as high as $89 \%$. The minimum force required to dislodge plantlets attached to rocky substrates reached $23.8 \mathrm{~N}$, which would potentially allow many plantlets to overcome winter storms in the field. The ability of rocky substrates to retain seedlings increased with their complexity. The interstitial spaces between rocks provided appropriate microsites for seedling settlement, as seeds were successfully retained and a suitable substrate for anchorage was available.

Adhesive root hairs allowed fast and strong seedling anchorage to consolidated substrates when the root system was not yet developed. This mechanism could favour plant recruitment on rocky substrates with respect to mobile ones, in contrast with traditional paradigms. Such an adaptation leads to hypothesize a new microsite driven bottleneck in $P$. oceanica seedling survival linked to substrate features. 